Results Over the 10-year period, there were 339 (5.6\%) deaths out of 6101 admissions to the PICU. 67 (19.8\%) out of a total of 339 deaths were associated with RVIs. Patients with RVI-associated mortality had a median age of 3 years (Q1 - Q3, 0 - 8). The majority were male $(n=38,56.7 \%) .23$ $(34.3 \%)$ of the patients were born preterm and $42(62.7 \%)$ had co-morbid conditions. Influenza (22.7\%), adenovirus (17.3\%), respiratory syncytial virus (RSV) (16\%) and rhinovirus $(16 \%)$ were the most common viruses isolated. Eight patients (11.9\%) had RVI coinfection. The most common documented cause of death in this cohort of RVI-associated mortalities was viral and/or secondary bacterial infections (76.1\%) followed by cardiovascular causes (7.5\%). The median hospital length of stay prior to death was 8 days (Q1 - Q3, 3 - 15).

Conclusions The burden of RVI-associated mortality is high among critically ill children. These data on the burden and age-specific distribution of RVI-associated mortality in children are critical in informing infection prevention practices among high-risk groups and immunization public health policies for RVIs. Efforts to improve influenza vaccination coverage especially in children with comorbidities or history of prematurity could have a significant impact in reducing this burden.

\section{SCOPING REVIEW ON THE EFFECTIVENESS OF THE USE OF TECHNOLOGY FOR PSYCHOSOCIAL SCREENING OF ADOLESCENTS IN URBAN HEALTH CARE SETTINGS}

Rebecca Loh. Australia

\subsection{6/bmjpo-2021-RCPCH.231}

Background The number of adolescents presenting with psychosocial issues at urban hospital pediatric emergency departments have increased significantly since 2000. Early psychological intervention has been proven to prevent future disabilities. Consequences from missing or delayed diagnosis of psychosocial issues in adolescence include increased suicide incidences and potential harm from extensive and invasive investigations. However, there is limited time and resources to evaluate youth for psychosocial stressors in healthcare facilities (Emergency department, clinics). Commonly, medical concerns are addressed first. Furthermore, youths are reluctant to disclose threats to their mental and social well-being when reviewed.

Objectives The effectiveness of technology in psychosocial screening of adolescents in urban health care settings was examined. Specifically, the rate and scope of detection of psychosocial stressors. This would allow for better understanding on how mental health screening resources for adolescence should be allocated. Advancements in psychosocial technological screening can potentially prevent unnecessary consumption of healthcare resources.

Methods A scoping review was used to study scientific literature regarding screening methods for mental health disorders in adolescence. Arksey and O'Malley framework was used to review articles from electronic databases such as BMJ, Cochrane, EBM, Pubmed, Uptodate. The review included cohort and experimental studies of adolescents (12-18 years old) from urban areas who were screened for mental health disorders using different screening tools. A secondary reviewer was asked to do further analysist of the articles during the second round of screening of the articles. Analytical research methods were then used to compare the detection rates and time taken to detect mental health problems in adolescence, using the various screening tools, both online and face to face.

Results 113 search articles were identified. 79 articles were yielded to be utilised in the review. Journal articles were included in the review if they were in English, published after 2000, conducted in metropolitan areas. Youths included in the studies were those who sought help at healthcare facilities (bothemergency department or clinic) in densely populated cities with easy access to both healthcare facilities and technology. Screening tools studied included standalone screening specific softwares, internet based softwares, telephone applications. Most screening tools utilised questionnaires and 3 utilised games. Journal articles studied included 37 randomized controlled trials, 14 Meta-Analysis, 7 Systematic Review, 2 Cohort Studies.

59 articles reflected that online psychosocial tools reached a wider spectrum of youths. 43 indicated that number of youths willing to undergo screening increased when online psychosocial tests were utilised. Screening rates for psychosocial problems of the target population of adolescence increased $6 \%$, compared with traditional face-to-face interview methods. Furthermore, youths were more willing to share openly on an online platform according to 5 research articles. As such, identification of mental disorders in youths was faster and more accurate when an online platform was utilised. Because of that, disclosure rate of the psychosocial stressors increased by $17 \%$ when online screening tools were utilised.

Conclusions It can be concluded that online screening tools are more effective, with regards to speed and scope of detection of mental health disorders amongst adolescence living in urban areas.

\section{RISK FACTOR AND OUTCOME OF ACUTE KIDNEY INJURY AMONG CRITICALLY ILL CHILDREN}

Wun Fung Hui, Vivian Pui Ying Chan, Kam Lun Hon, Man Hong Poon. Hong Kong

\subsection{6/bmjpo-2021-RCPCH.232}

Background Acute kidney injury (AKI) is an independent predictor of morbidity and mortality among critically ill children. However, epidemiological data in Asian paediatric populations remain scarce.

Objectives We presented the result of the interim analysis of an ongoing prospective cohort study on the epidemiology of AKI and electrolytes disturbances and their potential relationships with nephrotoxic medications (E-AKI-Drug) in a newly established paediatric intensive care unit (PICU).

Methods We enrolled all children aged 1 month to 18 years old admitted to the PICU of our hospital after June 2020. Those with pre-existing chronic kidney disease, impaired renal function for $\geq 3$ months, immediate post-renal transplant and short stay in PICU $<1$ day with no blood taking would be excluded. Children without a urinary catheter would be excluded from urine calculation. AKI would be defined using the KDIGO criteria. The medication records from 14 days prior to PICU admission to PICU discharge would be retrieved and reviewed by an independent pharmacist. The results of the initial 4 months of data collected would be presented. 
Results Altogether 62 patients with 63 episodes of admission were included for the analysis. Of these, $58.7 \%$ were male and the median (25th, 75th percentile) age was 6.1 (1.6, 12.7 ) years old. $49.2 \%$ of patients had a diagnosis of malignancy, $9.5 \%$ of them received bone marrow transplantation and $31.7 \%$ of patients were admitted post-operation. The overall incidence of AKI during PICU stay was 55.6\% using either the creatinine-based or urine output-based criteria (stage 1: 20.6\%, stage 2: $15.9 \%$ and stage 3: 19.0\%). Most patients experienced AKI on Day 1 of PICU admission using the creatine-based criteria (figure 1). Children with AKI had more types of electrolytes disturbances (5 types vs 3 types, $\mathrm{p}<0.01)$. Urine output and fluid overload on Day 1 of PICU admission were not significantly different between those with and without AKI. Risk factors for developing AKI during PICU admission included recipient of bone marrow transplantation (relative risk [RR with 95\% confidence interval]: $1.58[1.03,2.44])$, requirement of inotropic support (RR: $1.74[1.17,2.59]$ ) and non-invasive ventilation (RR: 1.76 [1.22, 2.55]), and a higher number of nephrotoxic medication exposure (RR: 1.20 [1.04, 1.38]) (table 1). $6.3 \%$ of patients required continuous renal replacement therapy. The overall mortality was $4.8 \%$. Patients with AKI had a longer PICU length of stay (4 vs 3 days, $\mathrm{p}=0.004)$ and hospitalization duration (23 vs 11 days, $\mathrm{p}=0.036)$ and a lower estimated glomerular filtration rate (eGFR) upon PICU discharge (136.1 vs $174.1 \mathrm{ml} / \mathrm{min} / 1.73$ $\left.\mathrm{m}^{2}, \mathrm{p}=0.012\right)$. Altogether $7.9 \%$ of patient were discharged from PICU with impaired renal function and 3.2\% of them were dialysis-dependent.

Conclusions AKI was commonly encountered among critically ill children. History of bone marrow transplantation, requirement of inotropic and non-invasive ventilatory support, and higher number of nephrotoxic medications were risk factors for AKI development. Those who had AKI were associated with a higher mortality, longer PICU and hospital stay and a lower eGFR on PICU discharge. A significant proportion of children with AKI were discharged with impaired renal function that warranted a long-term follow-up.

\section{ELECTROLYTES AND ACID-BASE DISTURBANCES: THE IMPACT AND RELATIONSHIPS WITH TUBULAR DYSFUNCTION AMONG CRITICALLY ILL CHILDREN}

Wun Fung Hui, Kam Lun Hon. Hong Kong

10.1136/bmjpo-2021-RCPCH.233

Background Electrolytes and acid-base disturbances are common yet largely ignored problems in critical care. Although closely related, the relationship between electrolytes disturbance and acute kidney injury (AKI) has not been extensively studied among critically children.

Objectives We presented the results of the interim analysis of an ongoing prospective cohort study on the epidemiology of acute kidney injury and electrolytes disturbances (E-AKI-Drug Study) in a newly established paediatric intensive care unit (PICU).

Methods All children aged 1 month to 18 years old admitted to the PICU of our hospital after June 2020 would be enrolled. Those with pre-existing chronic kidney disease, impaired renal function for $\geq 3$ months, immediate post-renal transplant and short stay in PICU $<1$ day with no blood taking would be excluded. Appropriate urinary investigations would be carried out if there were electrolytes disturbances. For children with more than two types of electrolytes disturbances, urine beta-2-microglobulin and amino acid profile would also be determined. AKI was defined using the KDIGO criteria. The results of the initial 4 months of data collected would be presented.

Results Altogether 63 episodes of admission were included for the interim analysis. $58.7 \%$ of the subjects were male and the median $(25$ th, 75th percentile) age was $6.1(1.6,12.7)$ years old. $49.2 \%$ of patients had a diagnosis of malignancy and 9.5\% received bone marrow transplantation. The overall incidence of AKI during PICU stay was 55.6\%. The median number of types of electrolyte disturbance was $4(2,5)$ types. Hypophosphataemia (85.5\%), hypocalacemia (77.4\%) and hypokalaemia (61.3\%) were the three most common types of electrolytes disturbances respectively. The incidence of metabolic acidosis and alkalosis were $90.3 \%$ and $35.5 \%$ respectively. Urinary wasting of potassium, phosphate and magnesium were common among children with hypokalaemia, hypophosphataemia and hypomagnesaemia, occurring in $25 \%$, $50 \%$ and $87.5 \%$ of the respective children (figure 1). Among children with more than two types of electrolytes disturbances, abnormal urinary beta-2-microglobulin level occurred in $64.7 \%$ of patient (median level $0.9[0.2,5.2] \mu \mathrm{g} / \mathrm{ml}$ ). The median degree of aminoaciduria was 23.1 (9.5, 47.6)\%. Children requiring inotropic $(5.0$ vs 3.0 types $\mathrm{p}<0.01)$ and ventilatory support (5.5 vs 4.0 types, $\mathrm{p}=0.008)$ and children with AKI $(5.0$ vs 3.0 types, $\mathrm{p}<0.01)$ had higher number of electrolytes disturbances. Besides, children with AKI had more hypernatraemia $(35.3 \%$ vs $10.7 \%, \mathrm{p}=0.036)$ and hypokalaemia (73.5\% vs $46.4 \%, p=0.029)$. Those with stage 3 AKI also had highest proportion of hyperphosphataemia compared to those with less severe or no AKI $(p=0.002)$. The number of types of electrolytes disturbances was associated with longer duration of ventilation $(\mathrm{r}=0.613, \mathrm{p}=0.011)$ and PICU length of stay $(r=0.567, p<0.001)$, as well as increased risk of PICU mortality (relative risk $4.3 \quad[95 \%$ confidence interval 1.4 , 12.7]).

Conclusions Electrolytes and acid-base disturbances were commonly encountered among critically ill children. Urinary wasting of the electrolytes and proximal tubular dysfunction were commonly observed among children with multiple electrolytes disturbances. The degree of electrolytes disturbances would contribute to longer ventilator days, PICU stay and even mortality, indicating a need to address the tubular health and its impact in critically ill children.

\section{THE APPLICATION OF EXTRACORPOREAL BLOOD PURIFICATION (EBP) IN CRITICALLY ILL CHILDREN}

Wun Fung Hui, Kam Lun Hon. Hong Kong

\subsection{6/bmjpo-2021-RCPCH.234}

Background EBP has been increasingly used to treat various conditions among critically ill patients and is frequently incorporated into the continuous renal replacement therapy (CRRT) circuit.

Objectives We reported our experience of applying EBP in a newly established Paediatric Intensive Care Unit

Methods The medical records of children requiring EBP in our hospital between $3 / 2019$ to $1 / 2021$ were reviewed 